\title{
SEMANTIC MOTION CONCEPT RETRIEVAL IN NON-STATIC BACKGROUND UTILIZING SPATIAL-TEMPORAL VISUAL INFORMATION
}

\author{
DIANTING LIU* and MEI-LING SHYU ${ }^{\dagger}$ \\ Department of Electrical and Computer Engineering \\ University of Miami, Coral Gables, FL 33124, USA \\ *d.liu4@umiami.edu \\ †shyu@miami.edu
}

\begin{abstract}
Motion concepts mean those concepts containing motion information such as racing car and dancing. In order to achieve high retrieval accuracy comparing with those static concepts such as car or person in semantic retrieval tasks, the temporal information has to be considered. Additionally, if a video sequence is captured by an amateur using a hand-held camera containing significant camera motion, the complexities of the uncontrolled backgrounds would aggravate the difficulty of motion concept retrieval. Therefore, the retrieval of semantic concepts containing motion in non-static background is regarded as one of the most challenging tasks in multimedia semantic analysis and video retrieval. To address such a challenge, this paper proposes a motion concept retrieval framework including a motion region detection model and a concept retrieval model that integrates the spatial and temporal information in video sequences. The motion region detection model uses a new integral density method (adopted from the idea of integral images) to quickly identify the motion regions in an unsupervised way. Specially, key information locations on video frames are first obtained as maxima and minima of the result of Difference of Gaussian (DoG) function. Then a motion map of adjacent frames is generated from the diversity of the outcomes from the Simultaneous Partition and Class Parameter Estimation (SPCPE) framework. The usage of the motion map is to filter key information locations into key motion locations (KMLs) that imply the regions containing motion. The motion map can also indicate the motion direction which guides the proposed "integral density" approach to locate the motion regions quickly and accurately. Based on the motion region detection model, moving object-level information is extracted for semantic retrieval. In the proposed conceptual retrieval model, temporally semantic consistency among the consecutive shots is analyzed and presented into a conditional probability model, which is then used to re-rank the similarity scores to improve the final retrieval results. The results of our proposed novel motion concept retrieval framework are not only illustrated visually demonstrating its robustness in non-static background, but also verified by the promising experimental results demonstrating that the concept retrieval performance can be improved by integrating the spatial and temporal visual information.
\end{abstract}

Keywords: Semantic retrieval; spatio-temporal; motion region detection; non-static background; integral density. 


\section{Introduction}

With the fast development of the Internet, more and more people search information on the Internet. Consequently, a number of text-based search engines appear on the Internet for topic and event searching tasks [1]. Although texts share certain correlation information with content data, searching for a multimedia content is not easy because multimedia data, as opposed to texts, needs more pre-processing steps to yield indices relevant for the query $[2,3]$. With the amount of online multimedia data increasing at an explosive speed, more challenges on data retrieval, browsing, searching and categorization arise. These challenges motivate many researchers to devote their efforts into the multimedia semantic retrieval area [4-6]. Specially, with the rapid advances of Internet and Web 2.0, in large multimedia databases, the traditional way of manually assigning a set of labels to a record, storing it, and matching the stored label with a query obviously is not feasible and effective. The successor methods, called content-based video retrieval approaches, are developed to quickly and automatically identify the semantic concepts and annotate the video sequences [7-11].

One of the key steps in content-based multimedia data analysis is automatic object detection which aims to segment a visual frame into a set of semantic regions and each region corresponds to an object that is meaningful to the human vision system such as a dog and a tree. However, the available information and the challenges of object detection from image data and video data are different. For example, the temporal information in video sequences enables us to utilize the moving object-level information for moving object detection. After years of development, many object detection models have been proposed with reasonably good performance in videos captured under controlled background. Nevertheless, little progress is achieved toward model robustness when dealing with videos with uncontrolled backgrounds, such as videos recorded by an amateur using a hand-held camera containing significant camera motion, background clutter, and changes in object appearance, scale, and illumination conditions. Figure 1 shows sample snapshots extracted from video clips recorded in an uncontrolled condition. These videos with uncontrolled backgrounds pose lots of challenges for multimedia retrieval over the Internet. This calls for the development of more advanced techniques for rapid processing and summarization. The drawbacks of the most existing techniques include the requirements of (1) static cameras or approximate compensation of camera motion; (2) foreground objects that move in a consistent direction or have faster variations in appearance than the background; and (3) explicit background models [12]. These requirements are mostly unrealistic and particularly questionable when an ego-motion happens, e.g. a camera that tracks a moving object in a manner such that the latter has very small optical flow, or the background is dynamic. In addition, background learning requires either a training set of "background-only" images [13] or batch processing (e.g. median filtering [14]) of a large number of "video frames". The latter must be repeated for each scene and is difficult for dynamic scenes where the background changes continuously. 

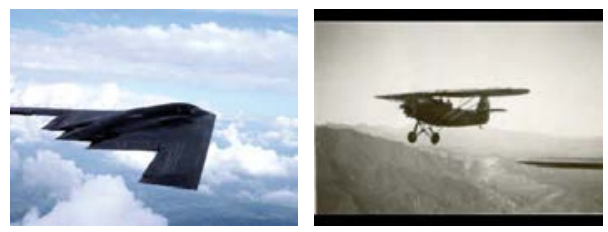

(a) Airplane-flying
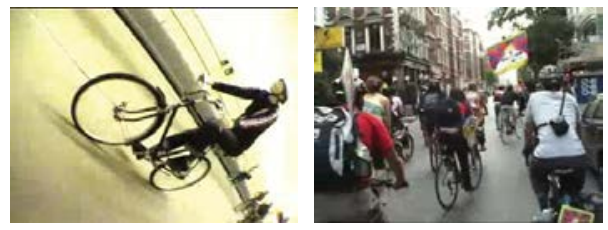

(c) Bicycling
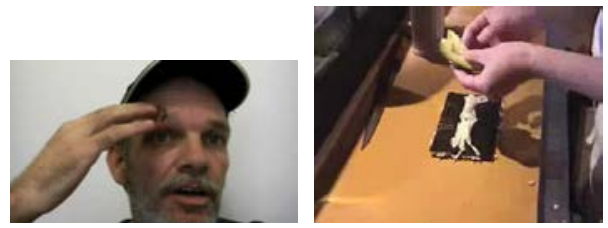

(e) Hand
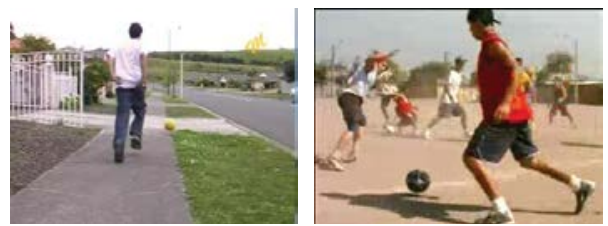

(g) Running
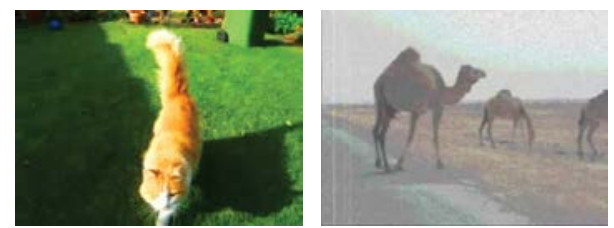

(b) Animal
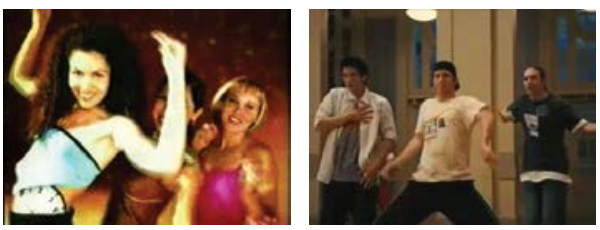

(d) Dancing
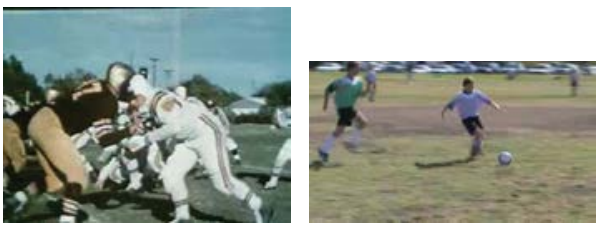

(f) Sports
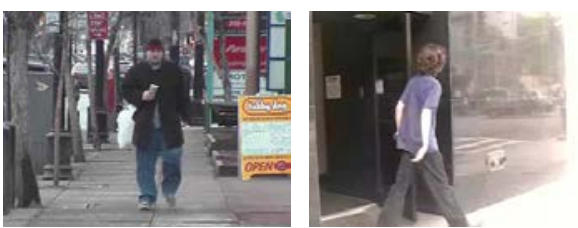

(h) Walking

Fig. 1. Snap-shots extracted from video clips recorded in uncontrolled condition of eight concepts from the TRECVID 2010 video collection.

It is found from psychological studies that a human vision system perceives external features separately [24] and is sensitive to the difference between the target region and its neighborhood. The high contrast is able to attract human's first sight more than the surrounding neighbors with low contrast [15]. Motivated by such findings, a motion region detection model is proposed in this paper. Our proposed model integrates spatial information locations (i.e. the yellow crosses shown in Fig. 2(a)) and temporal motion cues (i.e. the white and black zones shown in Fig. 2(b)) to find the locations that are rich in spatio-temporal information (i.e. the yellow crosses shown in Fig. 2(c)). After that, the proposed integral density method analyses the spatio-temporal information and identifies the motion region (i.e. the yellow bounding box shown in Fig. 2(d)). The motion regions can be viewed as a kind of motion information that may improve the semantic concept retrieval performance. 


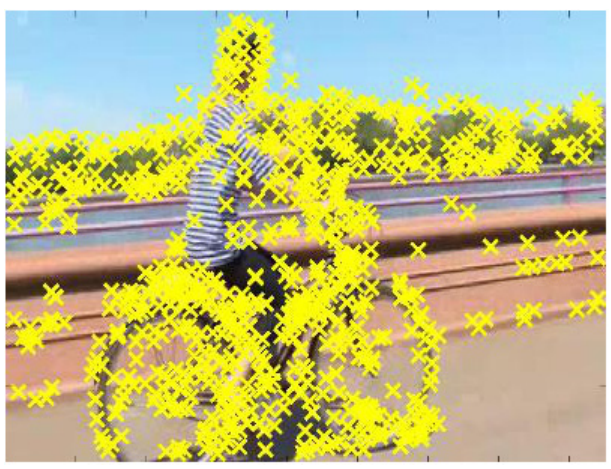

(a)

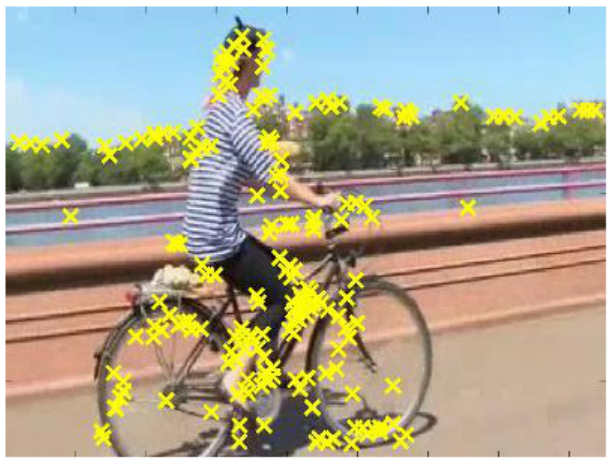

(c)

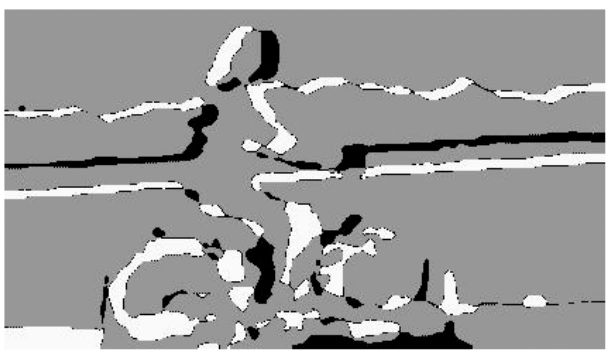

(b)

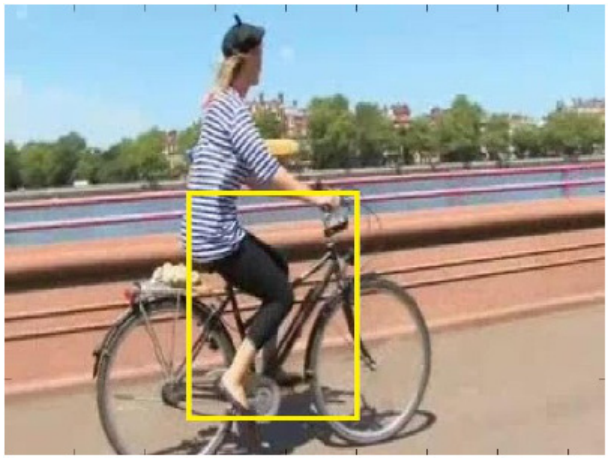

(d)

Fig. 2. Illustration of motion region detection. (a) Key information locations (yellow crosses) on a sample image. (b) Motion map of the sample image (white and black zones). (c) Key motion locations (KMLs) obtained via the motion map. (d) Motion region detected by the proposed integral density method from (c).

In this paper, a novel concept retrieval model that consists of a more general temporal consistency model and a conditional probability method is also proposed to enhance the semantic retrieval performance by taking into account of the semantic relevance among more than two shots.

The main contributions of this paper include:

(1) Define a new concept called key motion locations (KMLs) which are a subset of key information locations. Key information locations are obtained as the maxima and minima of the result of Difference of Gaussian (DoG) function.

(2) Propose a motion map based on the segmentation results generated from the Simultaneous Partition and Class Parameter Estimation (SPCPE) algorithm [23] in order to select the qualified key information locations as the key motion locations (KMLs). Such a motion map not only shows the motion areas, but also 
indicates the moving direction of the objects which help the identification of the motion region later.

(3) Propose an integral density method which is inspired by the idea of integral images in order to quickly and accurately detect the motion regions from KMLs.

(4) Present a semantic concept retrieval model to integrate global and local features.

(5) Utilize temporally semantic consistency among neighboring shots to re-rank the similarity scores to improve the retrieval performance.

The remainder of this paper is organized as follows. Section 2 discusses the related work in motion region detection and concept retrieval. The motion region detection model is presented in Sec. 3. Section 4 describes the proposed semantic retrieval model that combines the global and local features and utilizes temporally semantic consistency to enhance the semantic retrieval performance. Section 5 presents the experimental results and analyzes the performance from the detection and retrieval angles, respectively. Section 6 concludes the proposed semantic motion concept retrieval framework.

\section{Related Work}

Following the psychological findings, the detection of feature contrasts to trigger human vision nerves becomes the focus of many research studies. Such research led to the visual attention detection or salient object detection research areas. In [16], a conditional random field method which effectively combines multiple features such as multi-scale contrast, center-surround histogram, and color spatial distribution for salient object detection was proposed. When the saliency concept is moved from a static image domain to video sequences, the motion saliency which is also defined as attention due to motion [17] will dominate the frames. As a result, regardless of the visual texture in the scene, the human perceptual reactions will mainly focus on the motion contrast.

To further the research areas, many studies extended the research from the spatial attention to the temporal domain and the prominent motion becomes an important role. For instance, Mahadevan and Vasconcelos proposed an algorithm for spatiotemporal saliency based on a center-surround framework [12]. Their unsupervised algorithm combines spatial and temporal components of saliency in a principled manner, and is completely unsupervised. The main shortcoming of the work was its computational performance, so it is not applicable in real time. In [18], the authors proposed a backtrack-chain-updation split algorithm to distinguish two separate objects overlapped previously. Their proposed algorithm detected the split objects in the current frame, which are then used to update the overlapped ones in the previous frames in a backtrack-chain manner. In this way, more accurate temporal and spatial information of the semantic objects can be used for video indexing. Liu et al. [19] extended the work in [18] to handle more generalized overlapped situations though it has the constraint that the objects were finally split in the video sequences. Another 
study developed a spatio-temporal video attention detection technique that detects the attended regions corresponding to both interesting objects and actions in video sequences [20]. Their temporal attention model utilizes the geometric transformations between images as well as the interest point correspondences rather than the traditional dense optical fields. Furthermore, their model estimates the motion contrast by applying RANSAC (RANdom SAmple Consensus) on point correspondences in the scene, though its performance is greatly influenced by the results of point correspondences. Liu et al. [21] proposed a new framework that integrates the temporal information in [18] and the spatial clues used in [20] to achieve better performance in an unsupervised way.

In most of the existing video retrieval approaches, the relevance of a given shot with respect to a semantic concept is determined based on its own content and independently from its neighboring shots. The actual observation is that the temporally adjacent video segments (e.g. shots) usually have similar semantic concept for better semantic coherence and visual smoothness. This implies that those relevant shots matching a specific semantic concept or a query topic tend to gather in temporal neighborhoods or even appear next to each other consecutively. Based on the observed semantic consistency in the video sequences, we can assume that it could provide valuable contextual clues to video analysis and retrieval. With semantic consistency, one can make more informed prediction as to the relevance of the shot by considering the relevance of its neighboring shots, thus enhancing the overall performance of the predictions. Yang and Hauptmann presented a measurement of the temporally semantic consistency, namely transitional probability, to quantize the relevance of the semantic concept in two adjacent shots by calculating the conditional probability of the two shots [22]. This probability model was proved to be able to enhance the performance of video analysis and retrieval, though it only considers the relevance of two consecutive shots with the semantic concept.

\section{Motion Region Detection Model in Non-Static Background}

The optical flow method is considered one of the most commonly used algorithms in motion detection. Its basic idea is to calculate the motion contrast between visual pixels in the image, but it has some drawbacks. First, when multiple motion layers exist in the scene, optical flows at the edge pixels are noisy. Next, in texture-less regions, optical flows may return error values. In the paper, a fast object segmentation method called SPCPE (Simultaneous Partition and Class Parameter Estimation) is first employed to approximately segment the frame into background and foreground. Then the difference between the SPCPE results of the adjacent frames is calculated to generate a "motion map". This motion map is used to filter the key information locations obtained from the result of applying the Difference of Gaussian (DoG) function in the scale space to a series of smoothed and re-sampled videos frames [26]. Finally, the integral density method is utilized to identify the motion 


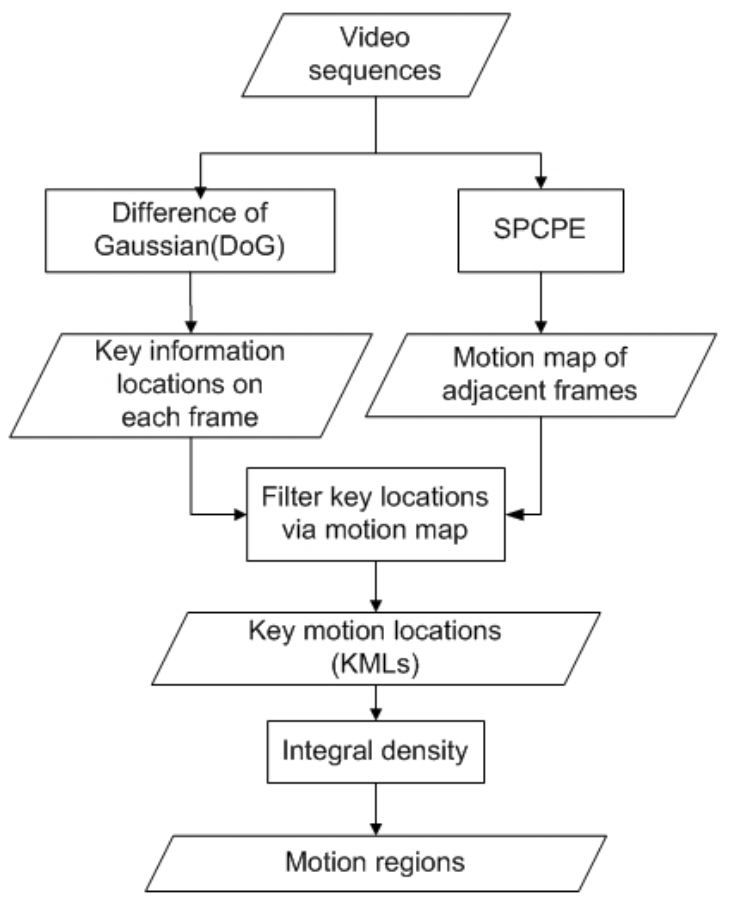

Fig. 3. The proposed motion region detection model.

regions where the density of key motion locations (KMLs) is high. Our proposed motion region detection model is presented in Fig. 3.

\subsection{Biological motivation}

Extensive psychophysical studies have shown that these mechanisms can be driven by a variety of features including intensity, color, orientation, motion, etc. The local feature contrast plays a predominant role in the perception of saliency. Neurophysiological experiments on primates have also shown that neurons in the middle temporal (MT) visual area compute local motion contrast with center-surround mechanisms. It has, in fact, been hypothesized that such neurons underlie the perception of motion pop-out and figure-ground segmentation [25]. On the other hand, this evidence suggests that spatio-temporal saliency or foreground motion detection techniques which (1) rely on grouping of features by motion similarity to identify foreground objects or (2) require compensation of camera motion will have difficulties to match the performance of biological systems. The center-surround mechanisms of biological systems support the idea of "motion map" on measurements of local motion contrast. One of the advantages of the proposed motion region model in the paper is that our method does not need training samples or pre-build a "global background model" for testing instances. Motion region can be efficiently calculated 
using merely motion information between the adjacent frames and could immediately adapt to different kinds of unknown scenes. Also, the use of the proposed motion map makes the motion region detection model robust to the camera motion and dynamic background.

\subsection{Motion map generation}

In this section, the motion region is identified from the non-static background in an unsupervised manner or a bottom-up approach. The top-down approaches are taskdriven and need to know the prior knowledge of the target. In contrast to such approaches, the bottom-up ones are a stimuli-driven mechanism based on the human reaction to the external stimuli such as the prominent motion from the surroundings.

Figure 4 shows the comparison between the binary images and the SPCPE segmentation results on some sample frames. In the third row of this figure, the segmentation results generated by SPCPE from two adjacent frames are presented and they show that the pixels in the video frames are segmented into two classes (in white and black, respectively). The SPCPE algorithm starts with an arbitrary class partition and employs an iterative process to jointly estimate the class partition and its corresponding class parameters. Typically, we can assume that the content of the adjacent frames in a video sequence does not change much, and thus the estimation result of the two classes of the successive frames does not differ a lot. These can be demonstrated between Figs. 4(a) and 4(b), and between Figs. 4(e) and 4(f).

Based on such an assumption, we used the segmentation of the previous frame as an initial class partition for the next frame to significantly reduce the number of iterations for processing. The iteration is terminated when the number of pixels that changed the class label between the two iterations is less than a threshold. Please note that though Figs. 4(e) and 4(f) do not show the precise contours of the objects, the segmentation is considered to reflect the object-level information in the frame. Though Figs. 4(c) and 4(d) obtained using binary images can in some degrees represent object-level information, the difference of binary images shown in Fig. 5(a) contains too much noise and therefore it fails to show the motion of moving objects. On the other hand, the results from SPCPE can better indicate the motion of moving objects as given in Fig. 5(b).

Assume the white regions and black regions in Figs. 4(e) and 4(f) stand for class 1 and class 2, respectively. The gray area in Fig. 5(b) shows the pixels which do not change the class labels from Fig. 4(e) to Fig. 4(f). The white zones in Fig. 5(b) illustrate those pixels which change from class 1 to class 2 , and the black zones shows the pixels which change from class 2 to class 1 . Apparently, these white and black zones contain the contour information of the foreground and background, as well as the motion direction information of the foreground. Thus we define the white and black zones in Fig. 5(b) as the motion map of Figs. 4(e) and 4(f). Figs. 5(c) and 5(d) are the motion region detection results by using different motion maps (Figs. 5(a) and 5(b), respectively). It shows that SPCPE can keep the general object 


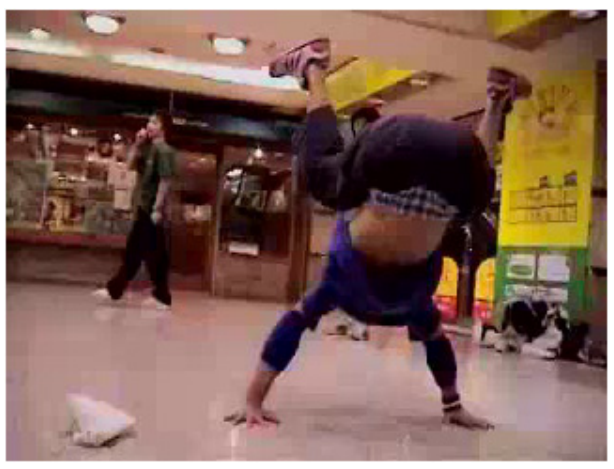

(a)

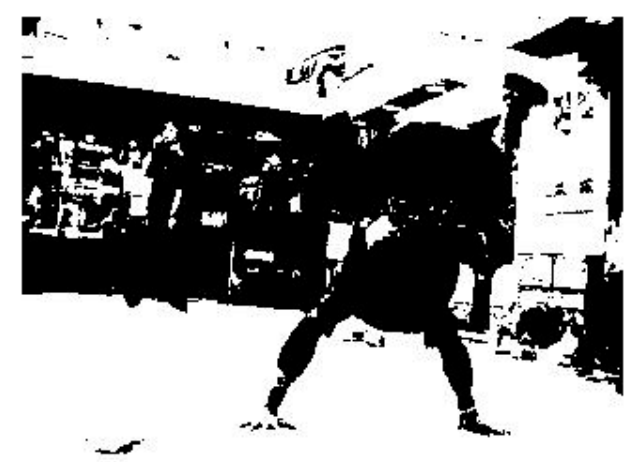

(c)

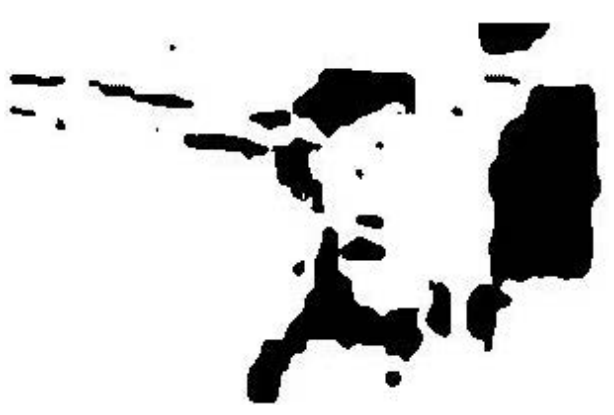

(e)

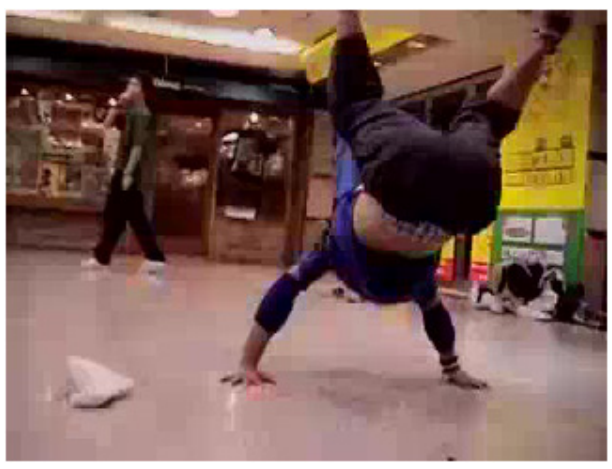

(b)

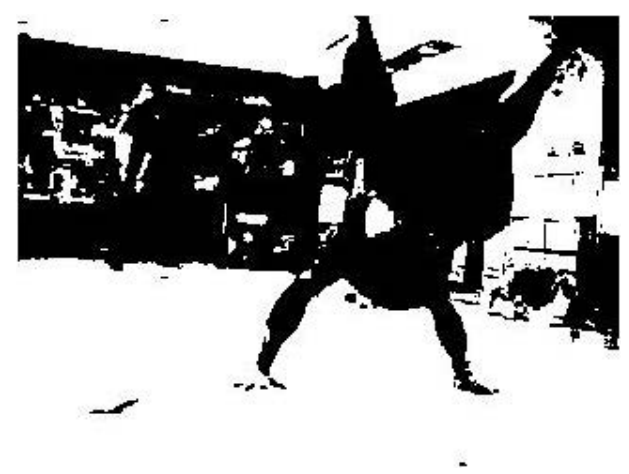

(d)
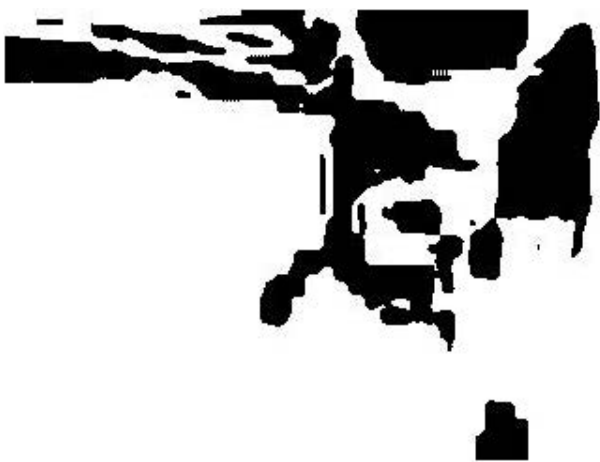

(f)

Fig. 4. Comparison of the binary images and SPCPE segmentation results on sample frames. (a) and (b) are adjacent sample frames containing camera motion, dancing person, and complex background, respectively. (c) and (d) are binary images converted from (a) and (b), respectively. (e) and (f) are the twoclass SPCPE segmentation results of (a) and (b), respectively. 


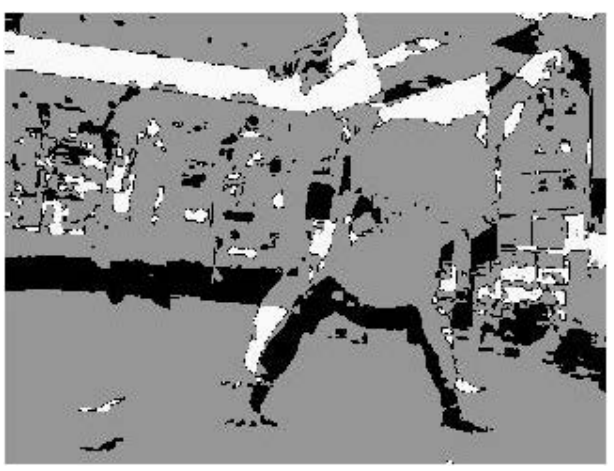

(a)

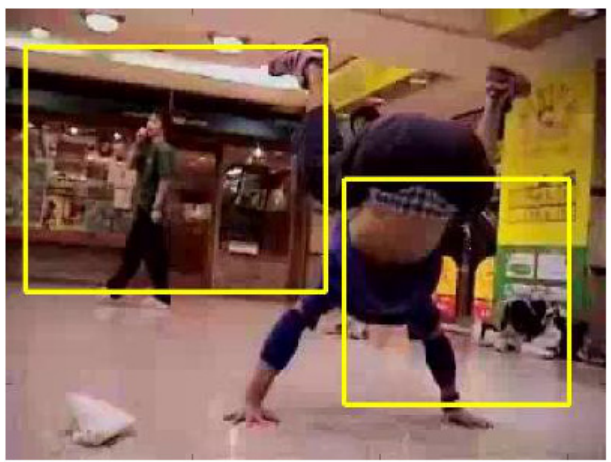

(c)

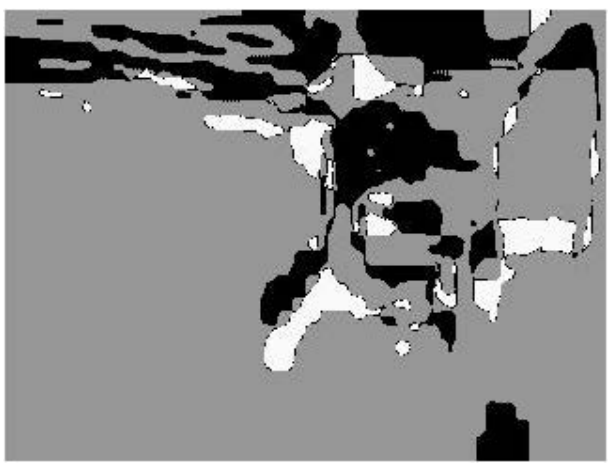

(b)

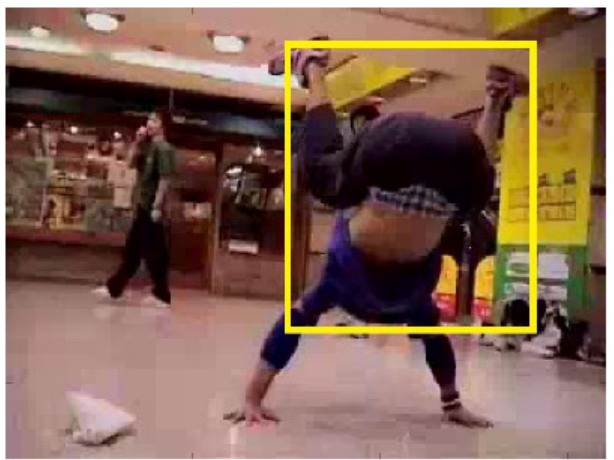

(d)

Fig. 5. Comparison of the motion maps and corresponding motion region detection results. (a) is the motion map generated from Figs. 4(c) and 4(d). (b) is the motion map created from Figs. 4(e) and 4(f). (c) and (d) are detection results via (a) and (b), respectively.

information while ignoring the detailed texture information, and therefore it is robust for generating a good motion map. In contrast, the binary images contain more detailed object contour information which may influence the quality of the motion map if the background in the frames contains many detailed textural information.

\subsection{Motion maps for identifying key motion locations}

In our proposed motion region detection model, we search the maxima and minima of the results of the DoG function when it is applied in the scale space to a series of smoothed and re-sampled frames to identify the key information locations [26].

In our framework, some key information locations describe the contours of the moving foreground; while the others describe the non-static background. On the basis of such an observation, the motion map generated in the previous step is used to remove the key information locations that are not located on the contour of the 
moving objects. In fact, we only keep those key information locations on the motion map since we consider them as motion related locations, and call them "key motion locations" (KMLs). For example, those KMLs in Fig. 2(c) are used to help find the motion regions shown in Fig. 2(d).

\subsection{Motion region detection}

With a batch of KMLs on hand, the next issue is how to group them into meaningful motion regions, which is a very time-consuming global search problem. To address this issue, the "integral density" method is proposed to quickly identify the motion regions. We define a motion region as one which has a higher density of KMLs than other regions and satisfies the direction constraint in the motion map. The idea of our proposed "integral density" method is adopted from the "integral images" in [27], since it allows fast implementation of the box type convolution filters.

Let the entry of an integral image $I_{\sum(\mathrm{x})}$ at a location $\mathbf{x}=(x, y)$ represent the sum of all pixels in the input image $I$ of a rectangular region formed by the point $\mathbf{x}$ and the origin. We then have the following:

$$
I_{\sum(\mathrm{x})}=\sum_{i=0}^{i \leq x} \sum_{j=0}^{j \leq y} I(i, j) .
$$

After $I_{\sum_{(\mathrm{x})}}$ is calculated, the sum of the intensities over any upright rectangular areas can be obtained in only four additions independent of their sizes.

Though the fast calculation idea is adopted from the integral image, it is the density of KMLs in the input image, instead of summarizing all pixels, that needs to be calculated. This leads to the definition of "integral density" in this paper. Through such a method, the region where the density of KMLs is high can be found effectively. In addition, such regions are considered to be highly related to the moving

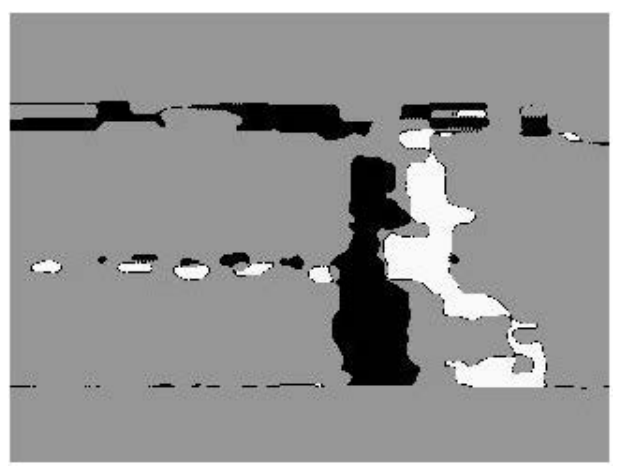

(a)

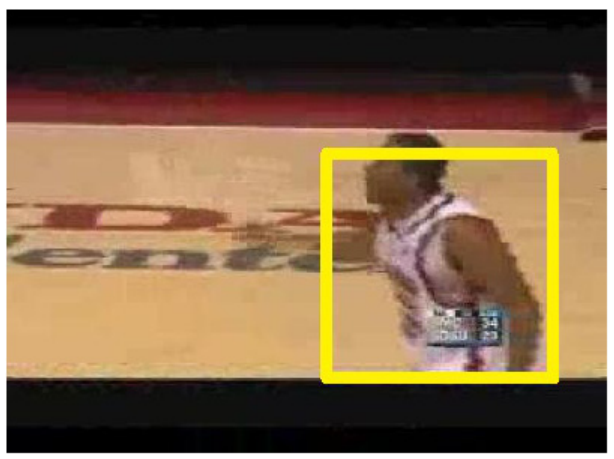

(b)

Fig. 6. The need of the proposed constraint for the integral density. (a) is the motion map of frame (b), which shows a correct motion region detection result under the given constraint. 
foreground. To ensure the bounding of a complete motion region, the constraint that the ratio of two motion zones (namely, the white zone and black zone) in the motion map is not high is imposed on each region. The rationale behind such a constraint is that ideally these two zones should have the same area, indicating the motion direction of the object. However, the question arises as how to decide such a ratio. Though the ratio threshold is set to two in this paper, it can be adjusted flexibly depending on the applications. Take Fig. 6(a) as the example. Since the white zone and black zone are separate, without such a constraint, only a half of the person in Fig. 6(b) where the density of the interest points is high will be bounded. Please note that the ratio does not depend on the speed of the object.

\section{Concept Retrieval Model}

Two new clues are introduced to enhance the semantic concept retrieval model. The first one is to extract local features from the motion region and integrate it with the global information for a better retrieval performance. The second one is to utilize the temporally semantic consistency information among shots in the conditional probability model to help re-rank the retrieval results.

\subsection{Semantic retrieval using global and local features}

The motivation is to utilize the information obtained from the motion region detection model in the manner that the local or object-level features are integrated with the commonly used global features for retrieval. Figure 7 presents the training phase of our proposed retrieval model. The training phase has the feature extraction module working on the motion regions, and the subspace training module working on the original frames.

To train the subspace in the proposed concept retrieval model, we adopt the representative subspace projection modeling (RSPM) algorithm [11]. Due to the facts that our proposed framework consists of two models, a subspace called the local subspace is trained for the local features extracted from the motion regions, and a subspace called the global subspace is trained for the global features extracted from the original video frames.

The testing phase of the proposed concept retrieval model is given in Fig. 8. The visual features are projected onto the subspace obtained in the training phase. That is, the local features extracted from the motion regions in the testing data set will be projected onto the local subspace for motion regions obtained in the training phase, and the global features extracted from the video frames in the testing data set will be projected onto the global subspace for the video frames obtained in the training phase. Each testing feature vector will be converted into a similarity score after the subspace projection. In order to combine the similarity scores from local and global subspaces, a fusion process is developed to generate a final similarity score to represent each video shot. In this paper, the logistic regression method is employed to 


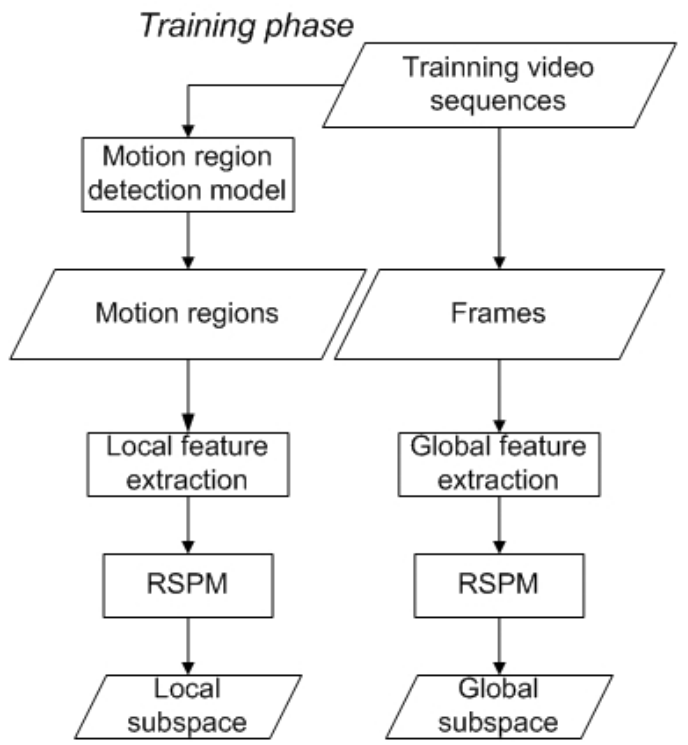

Fig. 7. Training phase of the proposed concept retrieval model.

\section{Testing phase}

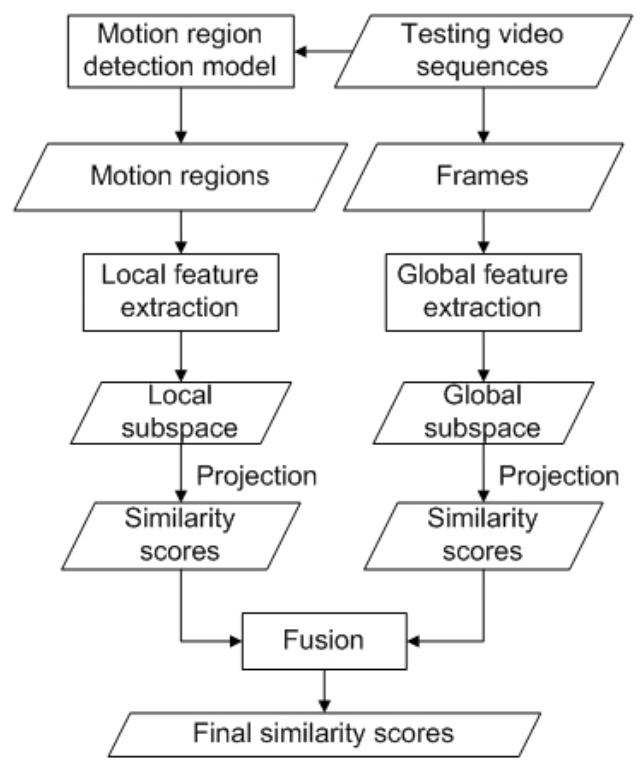

Fig. 8. Testing phase of the proposed concept retrieval model. 
fuse the global and local similarity scores from the different features. In the future, other fusion methods will be explored in our proposed model.

\subsection{Similarity score re-ranking using temporally semantic consistency}

In order to improve the retrieval performance, a conditional probability model is developed that provides a quantitative measurement of the semantic consistency to re-rank the retrieval results. This model considers the semantic relations among the neighboring shots, in particular, three contiguous shots in this study. Suppose $C_{t} \in$ $\{0,1\}$ is a binary variable indicating whether shot $t$ is relevant to semantic concept C. $P\left(C_{t}=1 \mid C_{t-1}=1, C_{t+1}=1\right)$ is defined as the conditional probability of shot $t$ being relevant to $C$ given that its previous shot $t-1$ and following shot $t+1$ are both relevant to $C$ (as shown in Eq. (1)).

$$
P\left(C_{t}=1 \mid C_{t-1}=1, C_{t+1}=1\right)=\frac{\sharp\left(C_{t}=1, C_{t-1}=1, C_{t+1}=1\right)}{\sharp\left(C_{t-1}=1, C_{t+1}=1\right)},
$$

where $\sharp\left(C_{t-1}=1, C_{t+1}=1\right)$ is the total number of shots whose preceding shot and succeeding shot are all relevant to concept $C$ in the training set, and $\sharp\left(C_{t}=1\right.$, $\left.C_{t-1}=1, C_{t+1}=1\right)$ is the total number of the three consecutive shot groups that are all relevant to concept $C$. In the same way, three other conditional probabilities are calculated as follows, considering the relevance between the shot and concept $C$.

$P\left(C_{t}=1 \mid C_{t-1}=1, C_{t+1}=0\right)$ is defined as the conditional probability of shot $t$ being relevant to concept $C$ given that its preceding shot $t-1$ is relevant; while the succeeding shot $t+1$ is irrelevant to concept $C$.

$$
P\left(C_{t}=1 \mid C_{t-1}=1, C_{t+1}=0\right)=\frac{\sharp\left(C_{t}=1, C_{t-1}=1, C_{t+1}=0\right)}{\sharp\left(C_{t-1}=1, C_{t+1}=0\right)},
$$

where $\sharp\left(C_{t-1}=1, C_{t+1}=0\right)$ is the total number of shots whose preceding shot is relevant to concept $C$; while the succeeding shot is irrelevant to concept $C$ in the training set, and $\sharp\left(C_{t}=1, C_{t-1}=1, C_{t+1}=0\right)$ is the total number of the three consecutive shot groups that the first two shots are relevant to concept $C$; while the third one is irrelevant to concept $C$.

In the same way, we may derive the following equations:

$$
\begin{gathered}
P\left(C_{t}=1 \mid C_{t-1}=0, C_{t+1}=1\right)=\frac{\sharp\left(C_{t}=1, C_{t-1}=0, C_{t+1}=1\right)}{\sharp\left(C_{t-1}=0, C_{t+1}=1\right)} \\
P\left(C_{t}=1 \mid C_{t-1}=0, C_{t+1}=0\right)=\frac{\sharp\left(C_{t}=1, C_{t-1}=0, C_{t+1}=0\right)}{\sharp\left(C_{t-1}=0, C_{t+1}=0\right)} .
\end{gathered}
$$

Equation (3) defines the conditional probability of shot $t$ being relevant to concept $C$ given that its preceding shot $t-1$ is irrelevant; while the succeeding shot $t+1$ is relevant to concept $C . \sharp\left(C_{t-1}=0, C_{t+1}=1\right)$ is the total number of shots 
whose preceding shot is irrelevant to concept $C$; while the succeeding shot is relevant to concept $C$, and $\sharp\left(C_{t}=1, C_{t-1}=0, C_{t+1}=1\right)$ is the total number of the three consecutive shot groups that the first shot is irrelevant to concept $C$; while the succeeding two shots are relevant to concept $C$. Equation (4) defines the conditional probability of shot $t$ being relevant to concept $C$ given that its preceding shot $t-1$ and the succeeding shot $t+1$ are both irrelevant to concept $C$. where $\sharp\left(C_{t-1}=\right.$ $\left.0, C_{t+1}=0\right)$ is the total number of shots whose preceding shot and the succeeding shot are irrelevant to concept $C$, and $\sharp\left(C_{t}=1, C_{t-1}=0, C_{t+1}=0\right)$ is the total number of the three consecutive shot groups that the first and the third ones are irrelevant to concept $C$; while the second one is relevant to concept $C$. Equations (1)-(4) provided a quantitative measurement of the semantic consistency in video sequences.

The probability of shot $t$ is relevant to concept $C$, also called the relevant score in this study, can be calculated as below.

$$
\begin{aligned}
P\left(C_{t}=1\right)= & P\left(C_{t}=1 \mid C_{t-1}=1\right) * P\left(C_{t-1}=1\right) \\
& +P\left(C_{t}=1 \mid C_{t-1}=0\right) * P\left(C_{t-1}=0\right),
\end{aligned}
$$

where $P\left(C_{t-1}=1\right)$ and $P\left(C_{t-1}=0\right)$ are the relevant and irrelevant scores of shot $t-1$ to concept $C . P\left(C_{t}=1 \mid C_{t-1}=1\right)$ in Equation (5) can be calculated in Eq. (6).

$$
\begin{aligned}
P\left(C_{t}=1 \mid C_{t-1}=1\right)= & P\left(C_{t}=1 \mid C_{t-1}=1, C_{t+1}=1\right) * P\left(C_{t+1}=1\right) \\
& +P\left(C_{t}=1 \mid C_{t-1}=1, C_{t+1}=0\right) * P\left(C_{t+1}=0\right),
\end{aligned}
$$

where $P\left(C_{t+1}=1\right)$ and $P\left(C_{t+1}=0\right)$ are the relevant and irrelevant scores of shot $t+1$ to concept $C$. Equation 6 then can be computed by using Eqs. (1) and (2). In the same way, $P\left(C_{t}=1 \mid C_{t-1}=0\right)$ in Eq. (5) can be calculated using Eq. (7) that is calculated via Eqs. (3) and (4).

$$
\begin{aligned}
P\left(C_{t}=1 \mid C_{t-1}=0\right)= & P\left(C_{t}=1 \mid C_{t-1}=0, C_{t+1}=1\right) * P\left(C_{t+1}=1\right) \\
& +P\left(C_{t}=1 \mid C_{t-1}=0, C_{t+1}=0\right) * P\left(C_{t+1}=0\right) .
\end{aligned}
$$

Please note that the relevant score of shot $t$ is predicted using the scores of its adjacent shots, aiming to improve the classification situation by utilizing the semantic consistency information in the video sequences.

\section{Experimental Results and Analysis}

We evaluate the performance of the proposed motion region detection model and the concept retrieval model on a subset of the TRECVID 2010 video collection [28]. The subset contains ten concepts which involve motion information as shown in Table 1. Some shots are multi-labeled. For example, a shot can be annotated as both "running" and "sports". 
Table 1. Data set parameters of the experiments.

\begin{tabular}{rcrr}
\hline $\begin{array}{c}\text { Concept } \\
\text { ID }\end{array}$ & Concept & $\begin{array}{c}\text { \# of shots } \\
\text { in training }\end{array}$ & $\begin{array}{c}\text { \# of shots } \\
\text { in testing }\end{array}$ \\
\hline 4 & Airplane-flying & 83 & 113 \\
6 & Animal & 687 & 1069 \\
13 & Bicycling & 79 & 55 \\
38 & Dancing & 390 & 250 \\
59 & Hand & 759 & 287 \\
100 & Running & 245 & 116 \\
107 & Sitting down & 1555 & 536 \\
111 & Sports & 607 & 839 \\
127 & Walking & 1067 & 412 \\
128 & Walking or running & 2145 & 766 \\
& Total & 7617 & 3604 \\
\hline
\end{tabular}

\subsection{Performance of the motion region detection model}

To form the experimental data set, a reference key frame for each shot is kept, assuming that the reference key frame represents the content of the shot. In the proposed motion region detection model and the semantic concept retrieval model, four extra frames around the reference key frame in each shot are extracted for the purpose of calculating the motion of the shots. In this study, the time interval between two frames is set to $0.2 \mathrm{~s}$. Such a value can be adjusted or adaptively computed based on the motion speed in the shot in our future work. Furthermore, to achieve fast computation, the minimum motion region size is set to 0.4 times of the shorter dimension size of the frame. This assumes that a small region only includes a part of a moving object. Some examples of motion region detection results in the data set are provided in Figs. 9-15.

Without considering the temporal motion information in the video sequences, there can be a large number of key information locations which represent a rich texture information area from the spatial angle, but some of them are considered noise (as shown in (a) of Figs. 9-15). As the result of applying our proposed key information location filtering via the motion map (in (c) of Figs. 9-15 obtained from the SPCPE algorithm), it can be clearly seen that the resulting key motion locations (KMLs) are able to keep the spatio-temporal information which is suitable for the motion region detection purpose (as shown in (d) of Figs. 9-15). The detection results from the temporal model of [20] are given in (f) of Figs. 9-15. The reason why the model proposed in [20] fails in some cases is that the model is greatly influenced by the results of point correspondences. Though the new model proposed in this paper uses a similar strategy as in [20] to locate the key information locations, our proposed motion map removes those motion-unrelated information and the integral density method successfully gets the motion region by precisely analyzing the distribution of the KMLs. Finally, the effectiveness of our proposed motion region detection model is demonstrated via (e) of Figs. 9-15. 


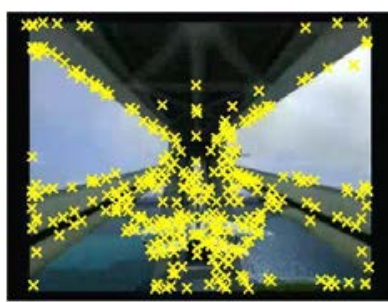

(a) Key information locations

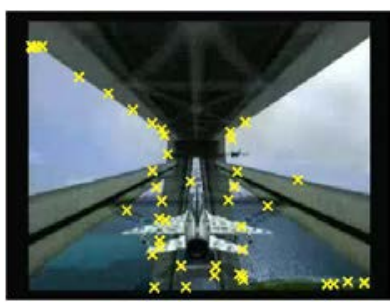

(d) Key motion locations (KMLs)

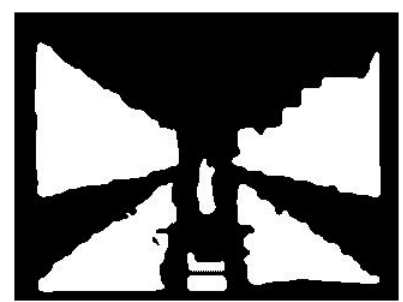

(b) SPCPE segmentation results

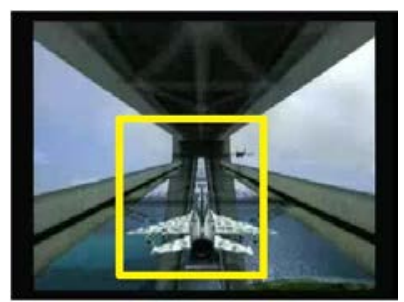

(e) Motion region by the proposed model

Fig. 9. Airplane flying.

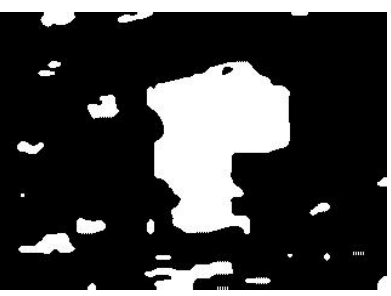

(b) SPCPE segmentation results

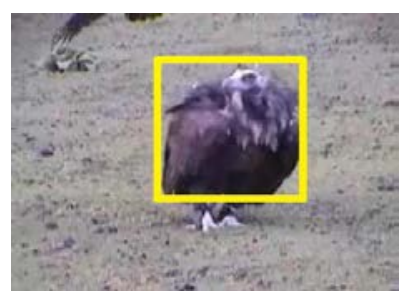

(e) Motion region by the proposed model

Fig. 10. Animal.

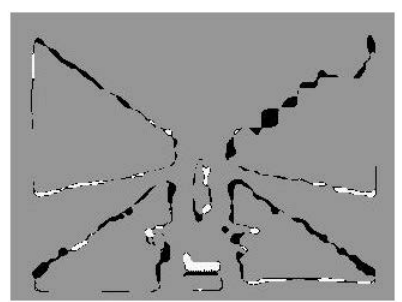

(c) Motion map

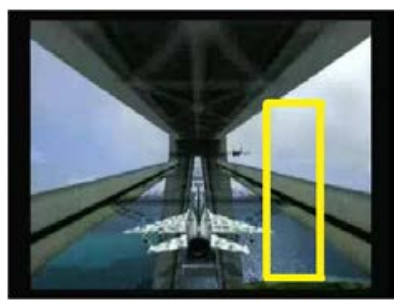

(f) Motion region by [20]

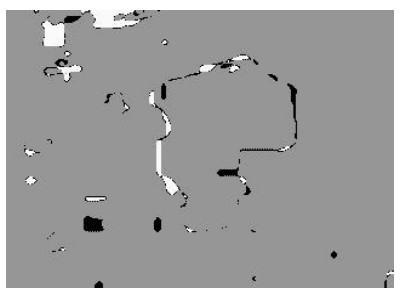

(c) Motion map

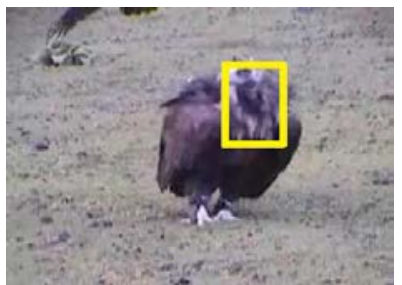

(f) Motion region by [20] (KMLs) 


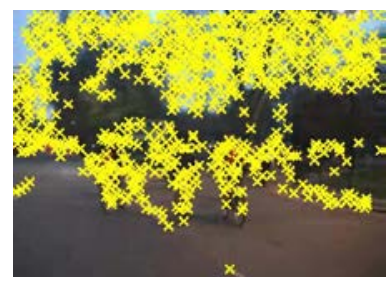

(a) Key information locations

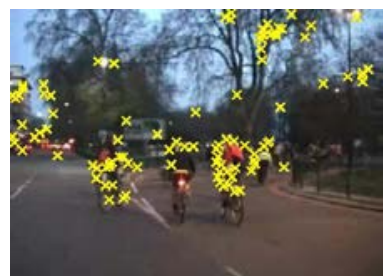

(d) Key motion locations (KMLs)

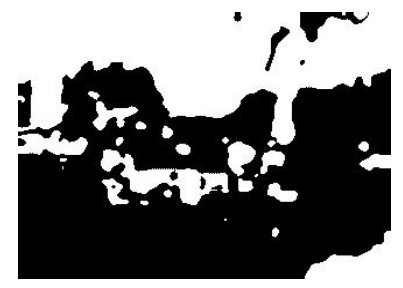

(b) SPCPE segmentation results

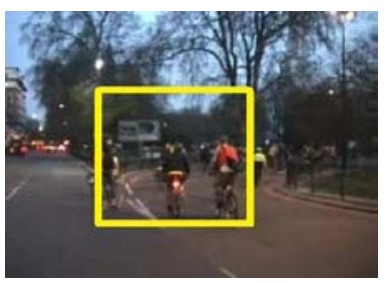

(e) Motion region by the proposed model

Fig. 11. Bicycling.

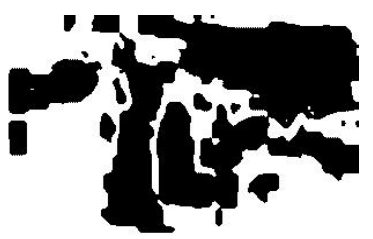

(b) SPCPE segmentation results

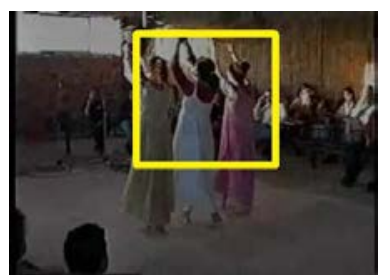

(e) Motion region by the proposed model

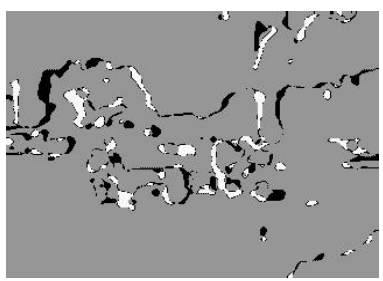

(c) Motion map

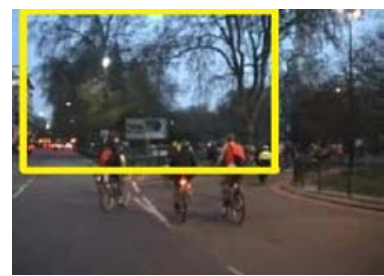

(f) Motion region by [20]

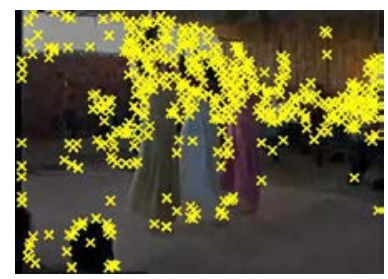

(a) Key information locations

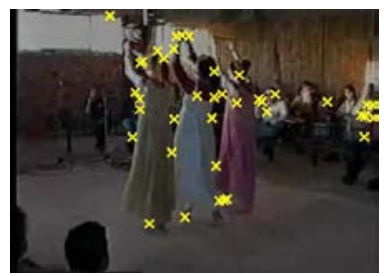

(d) Key motion locations (KMLs)

Fig. 12. Dancing.

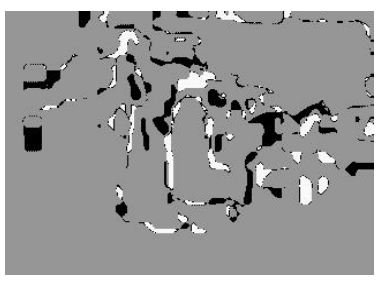

(c) Motion map

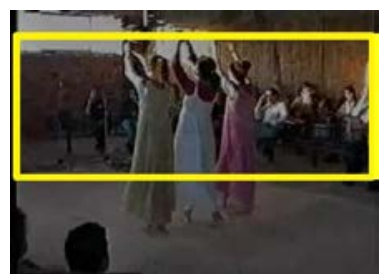

(f) Motion region by [20]

\subsection{Performance of the concept retrieval model}

Local features that describe the object-level texture of each shot are extracted from the detected motion regions. This may be complementary to the global information for the semantic concept retrieval. To verify this assumption, a set of comparable 


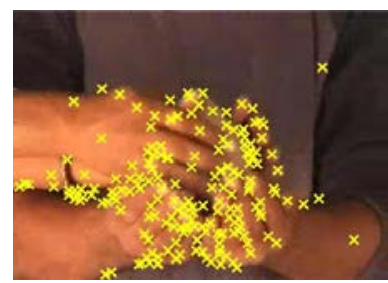

(a) Key information locations

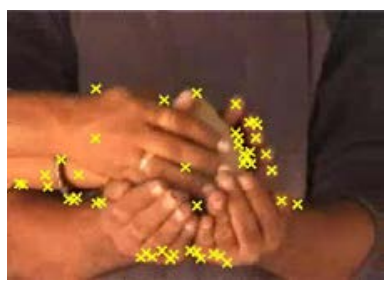

(d) Key motion locations (KMLs)

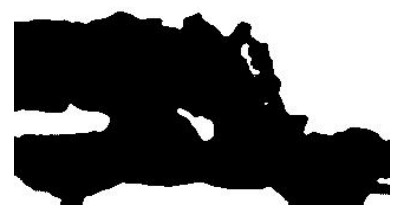

(b) SPCPE segmentation results

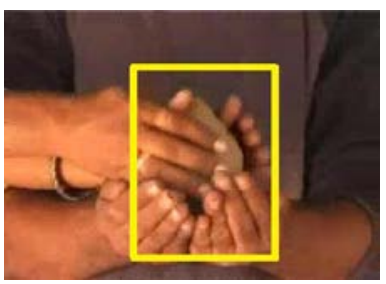

(e) Motion region by the proposed model

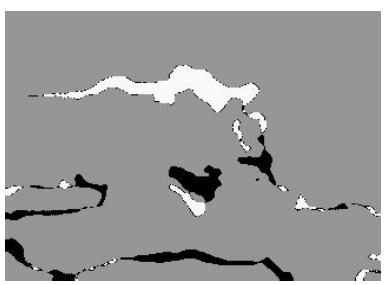

(c) Motion map

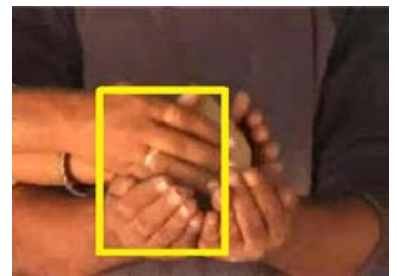

(f) Motion region by [20]

Fig. 13. Hand.

experiments is conducted in three data sets, namely the reference key frame (RF), multiple frames (MFA), and multiple frames plus motion regions (MFA+MR). The data set of the reference key frames is the same data set used in the motion region detection model. Four frames are extracted per shot around the reference key frame.

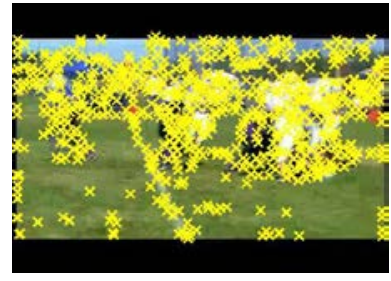

(a) Key information locations

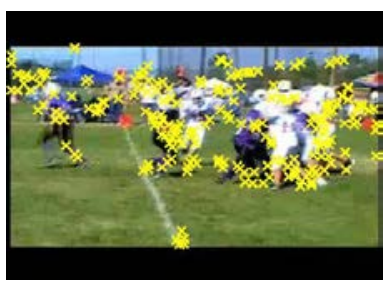

(d) Key motion locations (KMLs)

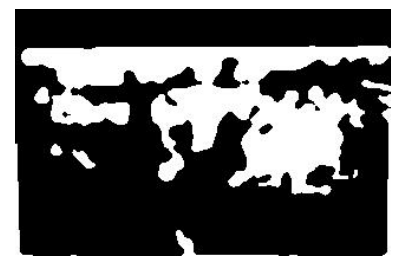

(b) SPCPE segmentation results

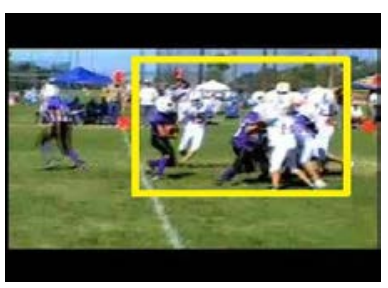

(e) Motion region by the proposed model

Fig. 14. Running.

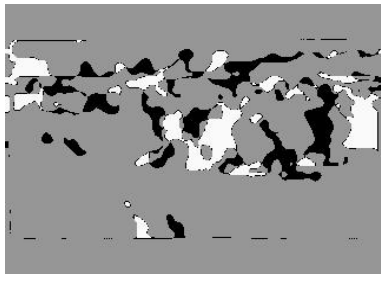

(c) Motion map

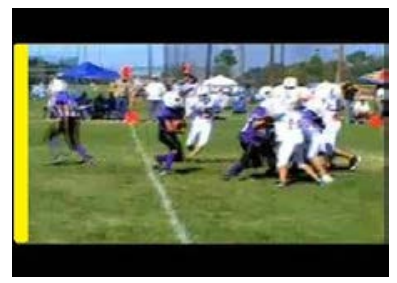

(f) Motion region by [20] 


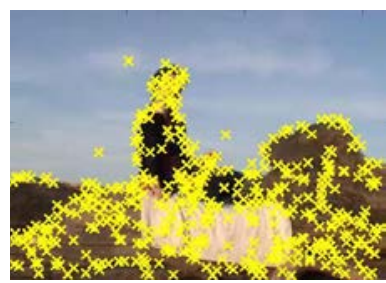

(a) Key information locations

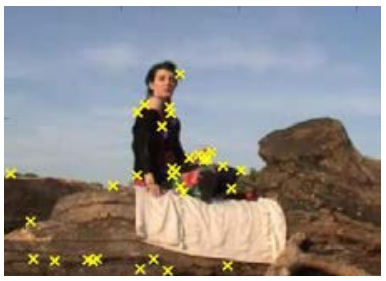

(d) Key motion locations (KMLs)

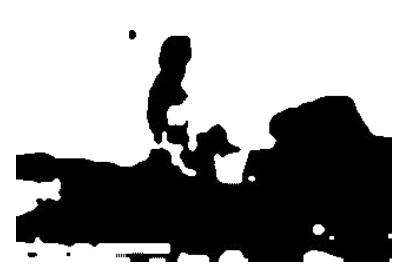

(b) SPCPE segmentation results

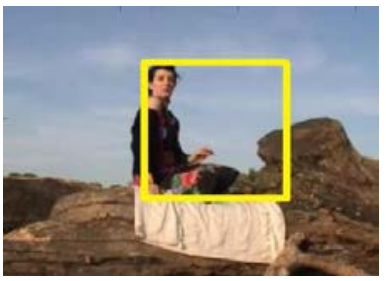

(e) Motion region by the proposed model

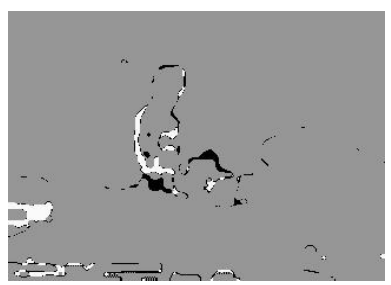

(c) Motion map

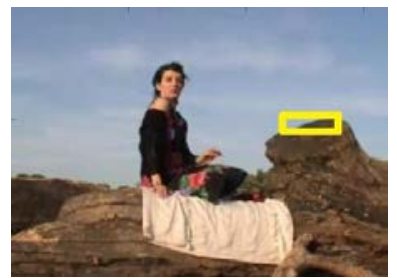

(f) Motion region by [20]

Fig. 15. Sitting down.

Including the reference key frame, each shot is represented by five frames which consist of the MFA data set. In each frame, one or more motion regions (MR) may be detected. Motion regions detected in the MFA data set plus the MFA data set itself form the MFA+MR data set. The experimental design aims to check whether the motion region features can complement the global features to enhance semantic concept retrieval performance.

For the comparison purpose, two kinds of texture features (YCC and LBP) are extracted from each data set. For the YCC features, the frame or region is first converted to the YCC color space from the RGB color space, and then that frame or region is divided into nine blocks. Four values, namely the mean, variance, skewness, and Kurtis, are calculated on the $\mathrm{Y}, \mathrm{Cb}$, and $\mathrm{Cr}$ components, respectively. We also calculate the mean, variance, skewness, and Kurtis values on the $\mathrm{Y}, \mathrm{Cb}$, and $\mathrm{Cr}$ components of the global frame, and hence there are totally 120 features extracted from each frame or region. LBP (Local Binary Pattern) is a texture operator that labels the pixels of a frame or region by threshold the neighborhood of each pixel and considers the result as a binary number. After summarizing the binary numbers, 59 LBP features are used to represent the frame or region.

Since there are similarity scores from multiple frames (MFA) and multiple frames and motion region $(\mathrm{MFA}+\mathrm{MR})$, the Logistic regression method is adopted to fuse the multiple similarity scores. Hence, the multi-class issue is transferred into the binary class problem. This means that in the training phase, the one-again-all strategy is utilized. 
The mean average precision (MAP) is defined as the mean of the average precision (AP) of all queries, and is used as the criterion to evaluate and compare the performance of different approaches. Average precision (AP) is a popular measure that takes into account both recall and precision in the information retrieval field. Strictly speaking, the average precision is the precision averaged across all recall values between 0 and 1 . In practice, the integral is closely approximated by a sum over the precisions at every possible threshold value, multiplied by the change in recall. Let $k$ be the rank in the sequence of retrieved shots, $n$ be the number of retrieved shots, $P(k)$ be the precision at cut-off $k$ in the list, and $\Delta r(k)$ be the change in recall from items $k-1$ to $k$ [29]. AP is defined as shown in Eq. (8).

$$
A P=\sum_{k=1}^{n} P(k) \Delta r(k) .
$$

Table 2 and Table 3 present the MAP values on retrieving 10, 100, 1000, 2000, and all shots in the three data sets. In these two tables, RF means the reference key frame data set; MFA means the multiple-frame data set including the reference key frame and four extra frames; and MFA+MR is the union of MFA and motion-region data set, including multiple frames with the motion region obtained from the multiple frames. The two tables show the consistent retrieval results among the three data sets. That is, MFA generally outperforms RF at different numbers of the retrieval shots. This demonstrates that using multiple frames could provide more useful information to improve concept retrieval performance than using a single reference key frame. On the other hand, MFA+MR outperforms both MFA and RF on both sets of features. This verifies that the motion region has the concept-related information that can be utilized in semantic concept retrieval. When comparing the MAP values in the same data set between Table 2 and Table 3, the YCC and LBP return similar MAP values. Also, we observe that the proposed motion region

Table 2. Comparison of MAP for different numbers of retrieval shots between three data sets using YCC features.

\begin{tabular}{cccccc}
\hline Top & 10 & 100 & 1000 & 2000 & All \\
\hline RF & 0.4528 & 0.4657 & 0.3745 & 0.3400 & 0.3063 \\
MFA & 0.5484 & 0.5503 & 0.4147 & 0.3752 & 0.3460 \\
MFA + MR & $\mathbf{0 . 6 7 4 6}$ & $\mathbf{0 . 6 1 0 3}$ & $\mathbf{0 . 4 3 5 5}$ & $\mathbf{0 . 3 9 6 8}$ & $\mathbf{0 . 3 6 9 1}$ \\
\hline
\end{tabular}

Table 3. Comparison of MAP for different numbers of retrieval shots between three data sets using LBP features.

\begin{tabular}{cccccc}
\hline Top & 10 & 100 & 1000 & 2000 & \multicolumn{1}{c}{ All } \\
\hline RF & 0.4929 & 0.5287 & 0.4370 & 0.4079 & 0.3915 \\
MFA & 0.5316 & 0.5541 & 0.4729 & 0.4437 & 0.4281 \\
MFA + MR & $\mathbf{0 . 6 2 0 9}$ & $\mathbf{0 . 6 0 3 4}$ & $\mathbf{0 . 4 9 8 3}$ & $\mathbf{0 . 4 6 5 9}$ & $\mathbf{0 . 4 5 0 1}$ \\
\hline
\end{tabular}


detection model indeed effectively identifies the motion region in the frame as shown in Figs. 9-15.

To evaluate the proposed global/local semantic concept retrieval model, a set of experiments conducted to verify the effectiveness of the proposed temporal consistency re-ranking approach. The method ranks the similarity scores obtained in the above semantic retrieval model, then using the conditional probability achieved from the training data set to update the similarity scores of the testing shots. The second rows in Tables 4-9 present the re-ranking results that use the temporal consistency information to update the results in Tables 2 and 3. The values in the first rows in Tables 4-9 are from Tables 2 and 3 for the comparison purposes. The experimental results show that the temporal consistency information extracted from the video sequences can help improve the final retrieval results, specially the MAP at top 10 having $10-25 \%$ increasing averagely in Tables $4-9$, that greatly improves the preliminary retrieval results.

Table 4. Comparison of MAP w/o and w/ temporal consistency (TC) applied on RF data set using YCC features.

\begin{tabular}{cccccc}
\hline Top & \multicolumn{1}{c}{10} & \multicolumn{1}{c}{100} & \multicolumn{1}{c}{1000} & \multicolumn{1}{c}{2000} & All \\
\hline No TC & 0.4528 & 0.4657 & 0.3745 & 0.3400 & 0.3063 \\
With TC & $\mathbf{0 . 6 0 6 3}$ & $\mathbf{0 . 5 1 7 9}$ & $\mathbf{0 . 4 4 2 1}$ & $\mathbf{0 . 4 1 4 1}$ & $\mathbf{0 . 3 8 6 5}$ \\
\hline
\end{tabular}

Table 5. Comparison of MAP w/o and w/ temporal consistency (TC) applied on MFA data set using YCC features.

\begin{tabular}{cccccc}
\hline Top & \multicolumn{1}{c}{10} & \multicolumn{1}{c}{100} & 1000 & \multicolumn{1}{c}{2000} & \multicolumn{1}{c}{ All } \\
\hline No TC & 0.5484 & 0.5503 & 0.4147 & 0.3752 & 0.3460 \\
With TC & $\mathbf{0 . 6 7 9 4}$ & $\mathbf{0 . 5 8 6 2}$ & $\mathbf{0 . 4 5 5 6}$ & $\mathbf{0 . 4 1 8 5}$ & $\mathbf{0 . 3 8 8 5}$ \\
\hline
\end{tabular}

Table 6. Comparison of MAP w/o and w/ temporal consistency (TC) applied on MFA+MR data set using YCC features.

\begin{tabular}{cccccc}
\hline Top & 10 & 100 & 1000 & \multicolumn{1}{c}{2000} & \multicolumn{1}{c}{ All } \\
\hline No TC & 0.6746 & 0.6103 & 0.4355 & 0.3968 & 0.3691 \\
With TC & $\mathbf{0 . 6 9 2 9}$ & $\mathbf{0 . 6 3 6 7}$ & $\mathbf{0 . 4 6 3 0}$ & $\mathbf{0 . 4 2 5 1}$ & $\mathbf{0 . 3 9 8 2}$ \\
\hline
\end{tabular}

Table 7. Comparison of MAP w/o and w/ temporal consistency (TC) applied on RF data set using LBP features.

\begin{tabular}{cccccc}
\hline Top & \multicolumn{1}{c}{10} & \multicolumn{1}{c}{100} & \multicolumn{1}{c}{1000} & \multicolumn{1}{c}{2000} & \multicolumn{1}{c}{ All } \\
\hline No TC & 0.4929 & 0.5287 & 0.4370 & 0.4079 & 0.3915 \\
With TC & $\mathbf{0 . 6 6 5 3}$ & $\mathbf{0 . 6 0 8 2}$ & $\mathbf{0 . 4 5 4 2}$ & $\mathbf{0 . 4 2 2 3}$ & $\mathbf{0 . 4 0 5 2}$ \\
\hline
\end{tabular}


Table 8. Comparison of MAP w/o and w/ temporal consistency (TC) applied on MFA data set using LBP features.

\begin{tabular}{cccccc}
\hline Top & 10 & 100 & 1000 & \multicolumn{1}{c}{2000} & All \\
\hline No TC & 0.5316 & 0.5541 & 0.4729 & 0.4437 & 0.4281 \\
With TC & $\mathbf{0 . 6 8 1 1}$ & $\mathbf{0 . 6 0 3 0}$ & $\mathbf{0 . 4 7 9 6}$ & $\mathbf{0 . 4 4 5 5}$ & $\mathbf{0 . 4 2 9 2}$ \\
\hline
\end{tabular}

Table 9. Comparison of MAP w/o and w/ temporal consistency (TC) applied on MFA + MR data set using LBP features.

\begin{tabular}{cccccc}
\hline Top & \multicolumn{1}{c}{10} & \multicolumn{1}{c}{100} & 1000 & \multicolumn{1}{c}{2000} & \multicolumn{1}{c}{ All } \\
\hline No TC & 0.6209 & 0.6034 & 0.4983 & 0.4659 & 0.4501 \\
With TC & $\mathbf{0 . 7 0 7 0}$ & $\mathbf{0 . 6 3 9 0}$ & $\mathbf{0 . 5 0 3 3}$ & $\mathbf{0 . 4 8 6 4}$ & $\mathbf{0 . 4 6 0 8}$ \\
\hline
\end{tabular}

\section{Conclusions}

This paper proposes a new semantic motion concept retrieval framework consisting of a motion region detection model and semantic retrieval model to analyze and utilize the spatio-temporal video sequence information. The framework is verified robust to the non-static background. Firstly, a motion map is generated from the SPCPE segmentation results to keep the motion related key information locations, called key motion locations (KMLs). Next, an integral density method is proposed to quickly and precisely identify the motion region by analyzing the density of the KMLs under the motion direction constraint generated by the motion map. Based on the detected motion regions, a new semantic retrieval model using global and local features are presented to effectively combine and fuse the texture information from the global features via the original frames and the local features from the motion regions. Finally, a re-ranking method that considers temporally semantic consistency information among continuous shots are proposed to further enhance the retrieval results. Experimental results show that our proposed motion region detection and semantic retrieval model achieves good performance in terms of the motion region detection and multimedia semantic retrieval.

\section{Acknowledgments}

We thank Anhui Huang for his technical suggestions on modeling the temporally semantic consistency into the conditional probability model.

\section{References}

[1] M.-L. Shyu, C. Haruechaiyasak and S.-C. Chen, Category cluster discovery from distributed WWW directories, Information Sciences 155(3) (2003) 181-197.

[2] M. Shyu, S. Chen, M. Chen and C. Zhang, A unified framework for image database clustering and content-based retrieval, in Proceedings of the 2nd ACM International Workshop on Multimedia Databases, Vol. 13 (2004), pp. 19-27. 
[3] M.-L. Shyu, S.-C. Chen, M. Chen, C. Zhang and K. Sarinnapakorn, Image database retrieval utilizing affinity relationships, in Proceedings of the 1st ACM International Workshop on Multimedia Databases, ACM, 2003, pp. 78-85.

[4] R. Datta, D. Joshi, J. Li and J. Z. Wang, Image retrieval: Ideas, influences, and trends of the new age, ACM Computing Surveys (CSUR) 40(2) (2008) 1-60.

[5] M. S. Lew, N. Sebe, C. Djeraba and R. Jain, Content-based multimedia information retrieval: State of art and challenges, ACM Transactions on Multimedia Computing, Communications and Applications 2(1) (2006) 1-19.

[6] L. Lin, C. Chen, M.-L. Shyu and S.-C. Chen, Weighted subspace filtering and ranking algorithms for video concept retrieval, IEEE Multimedia 18(3) (2011) 32-43.

[7] M. Chen, S.-C. Chen, M.-L. Shyu and K. Wickramaratna, Semantic event detection via temporal analysis and multimodal data mining, IEEE Signal Processing Magazine, Special Issue on Semantic Retrieval of Multimedia 23(2) (2006) 38-46.

[8] A. Hauptmann, M. Christel and R. Yan, Video retrieval based on semantic concepts, in Proceedings of the IEEE 96(4) (2008) 602-622.

[9] L. Lin and M.-L. Shyu, Effective and efficient video high-level semantic retrieval using associations and correlations, International Journal of Semantic Computing 3(4) (2009) 421-444.

[10] Z. Peng, Y. Yang et al., PKU-ICST at TRECVID 2009: High level feature extraction and search, in TRECVID 2009 Workshop, November 2009.

[11] M.-L. Shyu, Z. Xie, M. Chen and S.-C. Chen, Video semantic event/concept detection using a subspace-based multimedia data mining framework, IEEE Transactions on Multimedia, Special Issue on Multimedia Data Mining 10(2) (2008) 252-259.

[12] V. Mahadevan and N. Vasconcelos, Spatio-temporal saliency in dynamic scenes, IEEE Transactions on Pattern Analysis and Machine Intelligence 32 (2010) 171C177.

[13] Z. Zivkovic, Improved adaptive Gaussian mixture model for background subtraction, in Proceedings of the 17th International Conference on Pattern Recognition (2), IEEE, 2004, pp. 28-31.

[14] R. Cucchiara, C. Grana, M. Piccardi and A. Prati, Detecting moving objects, ghosts, and shadows in video streams, IEEE Transactions on Pattern Analysis and Machine Intelligence 25(10) (2003) 1337-1342.

[15] J. Duncan and G. Humphreys, Visual search and stimulus similarity, Psychological Review 96(3) (1989) 433-458.

[16] T. Liu, Z. Yuan, J. Sun, J. Wang, N. Zheng, X. Tang and H.-Y. Shum, Learning to detect a salient object, IEEE Transactions on Pattern Analysis and Machine Intelligence 33(2) (2011) 353-367.

[17] D. Mahapatra, S. Winkler and S. Yen, Motion saliency outweighs other low-level features while watching videos, in Proc. SPIE Vol. 6806, 2008, pp. 68060P.

[18] S.-C. Chen, M.-L. Shyu, C. Zhang and R. L. Kashyap, Identifying overlapped objects for video indexing and modeling in multimedia database systems, International Journal on Artificial Intelligence Tools 10(4) (2001) 715-734.

[19] D. Liu, M.-L. Shyu, Q. Zhu and S.-C. Chen, Moving object detection under object occlusion situations in video sequences, in Proceedings of the 2011 IEEE International Symposium on Multimedia, 2011, pp. 271-278.

[20] Y. Zhai and M. Shah, Visual attention detection in video sequences using spatiotemporal cues, in Proceedings of the 14th Annual ACM International Conference on Multimedia, 2006, pp. 815-824.

[21] D. Liu and M.-L. Shyu, Effective Moving Object Detection and Retrieval via Integrating Spatial-Temporal Multimedia Information, in Proceedings of the IEEE International 
Symposium on Multimedia (ISM2010), December 10-12, 2012, Irvine, California USA, pp. 364-371.

[22] J. Yang and A. G. Hauptmann, Exploring temporal consistency for video analysis and retrieval, in Proceedings of the 8th ACM International Workshop on Multimedia Information Retrieval, ACM, 2006, pp. 33-42.

[23] S. Sista and R. L. Kashyap, Unsupervised video segmentation and object tracking, Computers in Industry Journal 42(2) (2000) 127-146.

[24] A. Treisman and G. Gelade, A feature-integration theory of attention, Cognitive Psychology 12(1) (1980) 97-136.

[25] R. Born, J. M. Groh, R. Zhao and S. J. Lukasewycz, Segregation of object and background motion in visual area: Effects of micro-stimulation on eye movements, Neuron $\mathbf{2 6}$ (2000) $725-734$.

[26] D. G. Lowe, Distinctive image features from scale-invariant keypoints, International Journal of Computer Vision 60(2) (2004) 91-110.

[27] P. A. Viola and M. J. Jones, Rapid object detection using a boosted cascade of simple features, in Proceedings of the IEEE Conference on Computer Vision and Pattern Recognition, 2001, pp. 511-518.

[28] A. F. Smeaton, P. Over and W. Kraaij, Evaluation campaigns and TRECVid, in Proceedings of the 8th ACM International Workshop on Multimedia Information Retrieval, 2006, pp. 321-330.

[29] M. Zhu, Recall, Precision and Average Precision, University of Waterloo, Waterloo, ON, Canada, Working Paper 2004-09, 2004, Tech. Report. 